\title{
INFLUENCE OF AN IDENTIFIED DIMER VIBRATION ON THE EMISSION SPECTRUM OF I2.2 IPARACYCLOPHANE
}

\author{
W. GOLDACKER. D. SCHWEITZER. K.P. DINSE and K.H. HAUSSER \\ Max-Planck-Institut, Abteilung für Molekulare Physik, 6900 Heidelberg, Germany
}

Received 26 November 1979

\begin{abstract}
The emission spectrum of polycrystalline [2.2]paracyclophane shows a resolved vibronic structure with a $241 \mathrm{~cm}^{-1}$ progression at He temperatures. The dependence of the energy of this mode upon selective deuteration in combination with results from FIR and Raman spectra could be used to identify the mode as a torsional dimer vibration. The emission spectra could be simulated assuming a linear coupling of the torsional mode to the electronic transitions with coupling strengths of $S=10$ (fluorescence) and $S=13$ (phosphorescence). This corresponds to an equilibrium displacement of the benzene rings under electronic excitation by a torsional angle of $10.6^{\circ}\left(S_{1}\right)$ and $12.1^{\circ}$ $\left(T_{1}\right)$, in addition to the small torsion in the ground state $S_{0}$ by about $3^{\circ}$.
\end{abstract}

\section{Introduction}

Organic molecules in a low temperature rigid lattice usually exhibit a resolved intramolecular vibronic structure when observing their luminescence. Strong inhomogeneous optical line broadening due to different site energies or strong electronphonon coupling (EPC), however, can lead to a total loss of vibrational structure.

A first quantitative description of strong EPC as observed for impurity centres in anorganic crystals $[1,2]$ was given by Muto, Huang, Rhys and Pekar [3-5], and others $[6,7]$.

For emission from a molecular crystal, Haarer first used the theory of linear EPC to describe the $S_{1} \rightarrow S_{0}$ transition of the anthracene-PMDA complex [8]. This system is characterized by a broad unstructured emission in addition to a weak 0,0 -line. From the intensity ratio of the resolved 0,0-band to the total emission, the electron-phonon coupling strength was obtained. The energy of the coupled vibrational mode could be determined from a fit of the spectrum. From these values it was possible to calculate the shift of the equilibrium position under electronic excitation along the respective normal mode coordinate.
In our laboratory, phane molecules are currently investigated by optical spectroscopy and by ODMR. Phanes usually are dimers consisting of planar aromatic subunits coupled together by chemical bonds like methylene bridges. Luminescence from phanes in many cases is broad and structureless and without detectable 0,0 -lines [9-11]. This characteristic is found for phanes in highly diluted polycrystalline matrices as well as for trap emission from single crystals. The explanation of the optical linewidth by inhomogeneous site-broadening would therefore be unlikely.

In contrast, a change of the equilibrium nuclear positions under electronic excitation is expected from theoretical reasons [12], suggesting optical line broadening by strong EPC to a phane-cnerific vibrational mode.

A first estimate of vibrational energies from spectral widths leads to values of $300 \mathrm{~cm}^{-1}$ and less, values characteristic for skeletal modes. These values are expected when considering vibrations of the rigid monomers with respect to each other.

The assumption of EPC as a major source of optical line broadening in phane spectra could be tested for polycrystalline samples of [2.2] paracyclophane (see fig. 1), which show partially resolved 
vibrational structure, especially a long progression of a $241 \mathrm{~cm}^{-1}$ mode.

As will be shown below, the normal coordinate of this mode could be identified by selective deuteration and was found to be a torsion of the two subunits relative to each other.

The intensity distribution observed could be Fitted under the assumption of linear EPC, yielding values for the coupling strength $S$ of the fluorescence and phosphorescence transitions. In combination with the vibronic mode energy, equilibrium shifts under electronic excitation are obtainable.

\section{Experimental}

[2]]paracyclophane was crystallized from solution [13]. The isotopically different molecules investigated are given in fig. 1 .

The polycrystalline samples were investigated in a liquid He cryostate. Optical excitation was performed with a HBO 200 lamp in combination with a Schoeffel GM $2500.25 \mathrm{~m}$ monochromator and UV-R-310 plus UG 11 filters. Emission was detected with a Spex $14020.85 \mathrm{~m}$ monochromator and $R C A$ 31034-AO2 photomultiplier. Monochromator resolution was $3 \AA$.

The Raman spectrum was recorded at $5 \mathrm{~K}$. As an excitation source we used a single mode dye laser (Coherent 599-21) tuned to $5925 \AA$ with incident power of $50 \mathrm{~mW}$. Detection was performed with a Spex $14020.85 \mathrm{~m}$ double monochromator with holographic gratings and spatial filtering option together with a PAR/Ortec photon-counting system [14]. Raman lines could be recorded for frequencies larger than $5 \mathrm{~cm}^{-1}$. In order to increase the signal-to-noise ratio in the Raman spectrum, a single crystal of approximately $6 \times 5 \times 5 \mathrm{~mm}$ was used.
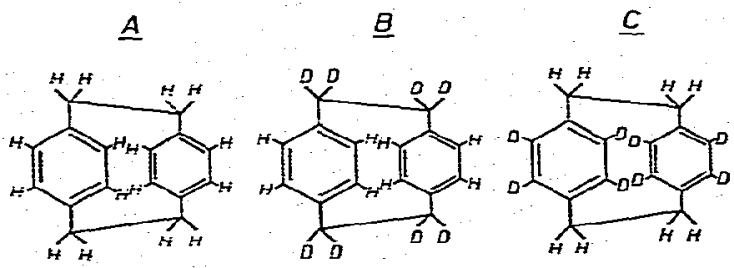

Fig. 1. Isotopically substituted [22]paracyclophane molecules.
For crystal preparation [2.2]paracyclophane (Frinton Laboratories) was recrystallized twice from chloroform. A saturated filtered solution was prepared in toluene at $65^{\circ} \mathrm{C}$ and after being kept for two days, the temperature was lowered at a cooling rate of $0.5^{\circ} \mathrm{C} /$ hour. Suitable seed crystals, obtained by this method, were replaced in an other accurately saturated toluene-solution at $65^{\circ} \mathrm{C}$. By keeping this solution at a cooling rate of $0.05^{\circ} \mathrm{C} / \mathrm{hour}$ for approximately 20 days, single crystals of reasonable size were grown.

The FIR spectra were kindly obtained by Bruker GmbH at a commercial spectrometer.

\section{Results}

\subsection{Optical spectr}

Fluorescence and phosphorescence spectra of all paracyclophane species show a vibronic progression superimposed on a broad band (fig. 2). (The spectrum of $B$, which is not shown, is nearly identical to the spectrum of A.) A prominent mode can be identified in all cases and the vibrational energies are compiled in table 1.

Table 1

Fluorescence and phosphorescence 0,0 transition energies of the various [2.2]paracyclophane species obtained at $1.2 \mathrm{~K}$. The phosphorescence 0,0 -line position is calculated using $\bar{v}_{\mathrm{t}}$ and the experimentally observed 0,2 -transition. The coupling strength $S_{z}$ used for the spectrum fit (see fig. 2) is given in combination with the coupled torsional mode energy $\bar{v}_{z^{-}}$. For the fit is further assumed that an additional monomer mode $\left(\bar{y}_{\mathrm{c}}=1600 \mathrm{~cm}^{-1}\right)$ is weakly coupled with strength $S_{c}$ to the electronic transition. The resulting equilibrium shift under electronic excitation is denoted by $\Delta Q$

Species \begin{tabular}{l}
$\begin{array}{l}0,0 \text {-band } \\
(0,2-b a n d) \\
\left(\mathrm{cm}^{-1}\right)\end{array}$ \\
\hline
\end{tabular}

fiuorescence

\begin{tabular}{lcllll} 
A & $30364(3)$ & $10.0(5)$ & $0.50(5)$ & $241(1)$ & 10.6 \\
B & & 10 & & $239(1)$ & \\
C & $30472(3)$ & $10.0(5)$ & $0.25(5)$ & $230(1)$ & 10.2 \\
phosphorescence & & & & \\
A & $24602(3)$ & $13.0(5)$ & $0.75(5)$ & $243(1)$ & 12.1 \\
B & $(24117(3))$ & & & & \\
C & $24643(3)$ & $13.0(5)$ & $0.70(5)$ & $230(1)$ & 11.6 \\
\hline
\end{tabular}




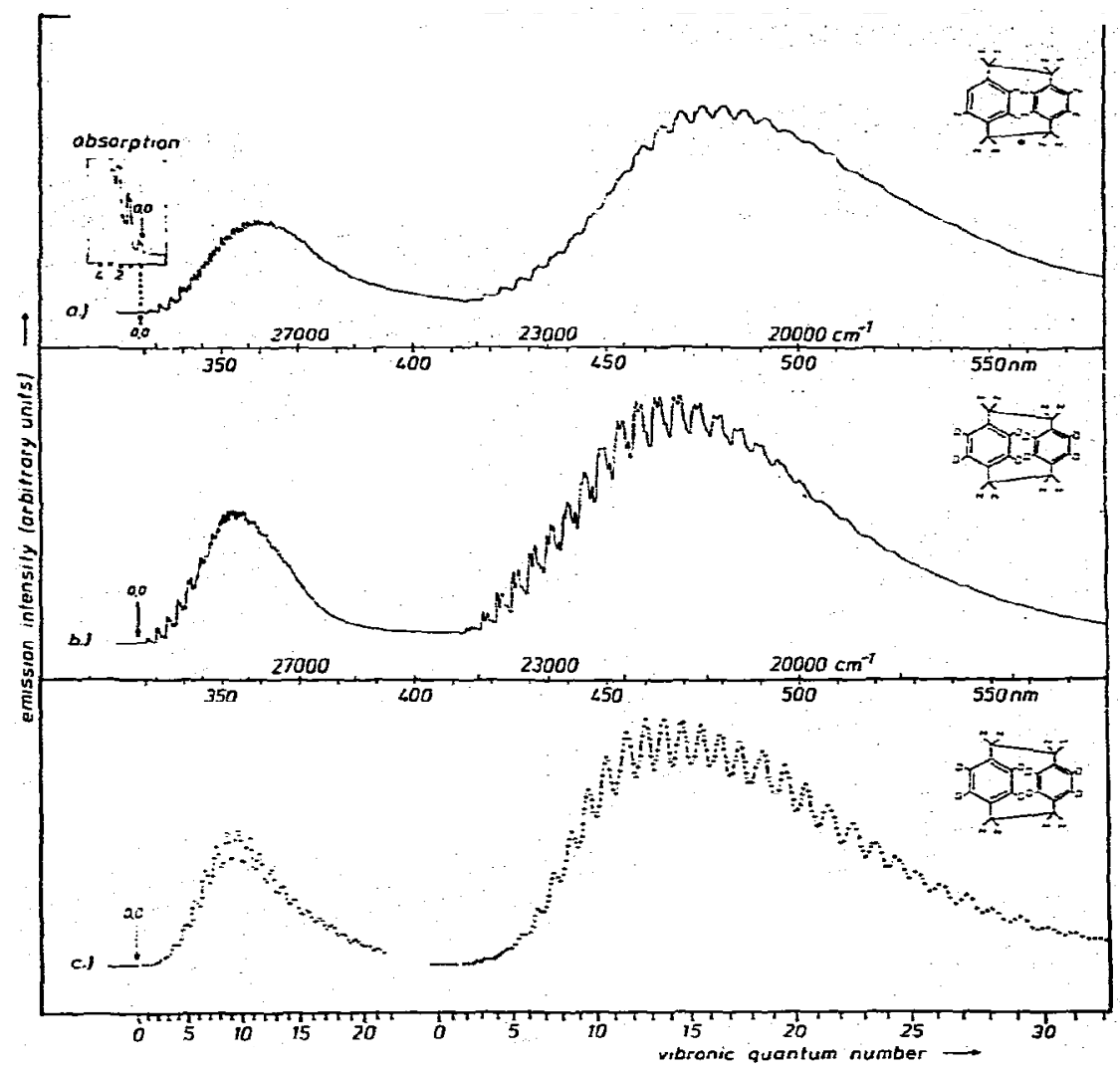

Fig. 2. Fluorescence and phosphorescence emission of $A(a)$ and $C(b)$ at $1.2 \mathrm{~K}$ obtained with $\lambda_{\text {exc. }}=313 \mathrm{~nm}$. The position of the weak 0,0 -fluorescence transition is verified by the position of the corresponding absorption line [see insert in (a)]. The simulated spectrum for species $C$ using the parameters given in table 1 is depicted in (c). The linewidth $E_{\mathrm{hw}}$ was chosen as $E_{\mathrm{hw}}=0.75 \hbar \omega_{\mathrm{r}}$. The vanishing structure in the experimental spectrum at the fluorescence maximum at variance with the calculated spectrum is probably due to the increasing intensitv of the additionally coupled low frequency modes, which were not included in the spectrum fit.

As expected, for a given species the values taken from fluorescence and phosphorescence spectra are identical within experimental error.

Additional vibronic bands [15] can be identified, but they show weaker progressions and are characterized by smaller energies $(\bar{v}=50(5), 80(3)$, $180(8) \mathrm{cm}^{-1}$ for species A).

Ron and Schnepp [16] had analyzed the absorption spectrum for species $A$ and measured the dominant vibrational mode energy as $237 \mathrm{~cm}^{-1}$. This value differs only by $1.5 \%$ from the value found in the fluorescence spectrum. The invariance of the normal mode frequency under electronic excitation from $S_{0}$ to $S_{1}$ is important when applying the linear approximation to the EPC theory (see below).

The intensity maximum in all emission spectra is shifted by about ten vibrational quanta with respect to the first observed band (not necessarily the 0,0-transition). The observed spectra therefore are examples of strong electron vibrational coupling.

Fig. 3 shows the Franck-Condon diagram for a coupling strength of $S=10$ together with a $T=0 \mathrm{~K}$ intensity distribution according to eq ( 7 ) below.

The identification of the 0,0 -line position of the 


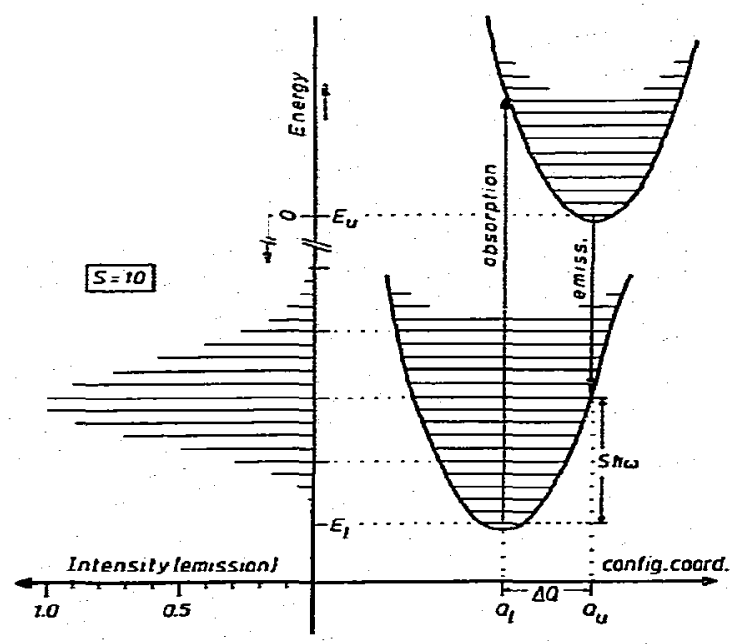

Fig 3 . The FC-diagram for a single harmonic oscillator linearly coupled to an electronic transition is shown on the right hand side. The displacement $\Delta Q$ of the normal coordinate equilibrium position is given for a coupling strength $S=10$. The calculated emission intensity distribution (left hand side) shows a maximum near the 0,10 -transition.

fluorescence of $A$ is performed by recording the absorption spectrum (see insert in fig. 2). The value found is in agreement with ref. [16].

\subsection{Identification of the prominent vibration}

The observed small isotopic dependence of the vibration energies (see table 1) can be used for a mode identification, when first the mode is ascribed to a low-energy dimer vibration.

The two most simple dimer modes in paracyclophane can be described as a breathing mode (motion of the subunits along the common molecular out-of-plane axis) and a torsional mode (torsion of the subunits about the out-of-plane axis) [17]. Deuteration (d) will in both cases reduce the mode frequency as compared with the non-deuterated species (nd). The reiative frequency reduction $R$ is given by

$R=h \omega^{\mathrm{d}} / h \omega^{\mathrm{ad}}-1=\left(\zeta^{\mathrm{nd}} / \xi^{\mathrm{d}}\right)^{1 / 2}-1$,

where $\bar{\zeta}$ is either the reduced mass (breathing mode) or the reduced moment of inertia (torsional mode).
In contrast to the reduced mass, which is independent of the position of deuteration, the moment of inertia change depends quadratically on the distance of the respective deuterium position from the torsional axis. For a ring deuteration the calculated $R$ (torsion) $=6 \%$ is much larger than $\boldsymbol{R}$ (breathing) $=2.5 \%$. The experimental value $R=5 \%$ is clearly only consistent with the assumption of a torsional mode. This is in agreement with a small reduction $R$ under methylene bridge deuteration.

\subsection{Ramian and IR spectra}

Evidence for a torsion of the paracyclophane subunits was already obtained from an analysis of $X$-ray reflexes $[17,18]$. A torsion of the benzene rings by an angle of about $3^{\circ}$ was calculated. Whether the torsion in the ground state is static or dynamic, i.e. whether the molecular symmetry is $D_{2}$ or $D_{2 \mathrm{~h}}$ could not be decided from these experiments. However, the investigation of the IR and Raman activity of the torsional mode can lead to a determination of the ground state symmetry: A vibration of $A_{u}$ symmetry is neither IR nor Raman active in $D_{2 h}$. For $D_{2}$ molecular symmetry one finds Raman activity, however.

The FIR and Raman spectra are shown in figs. 4 and 5. The torsional mode is only detectable in the Raman spectrum. We therefore conclude, that [22] paracyclophane is statically distorted in the

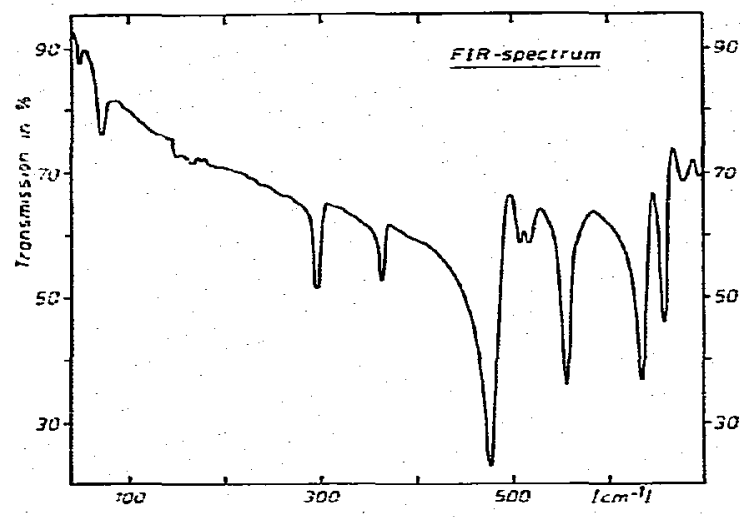

Fig. 4. FIR-spectrum of [2.2]paracyclophane (species C) obtained at room temperature. 


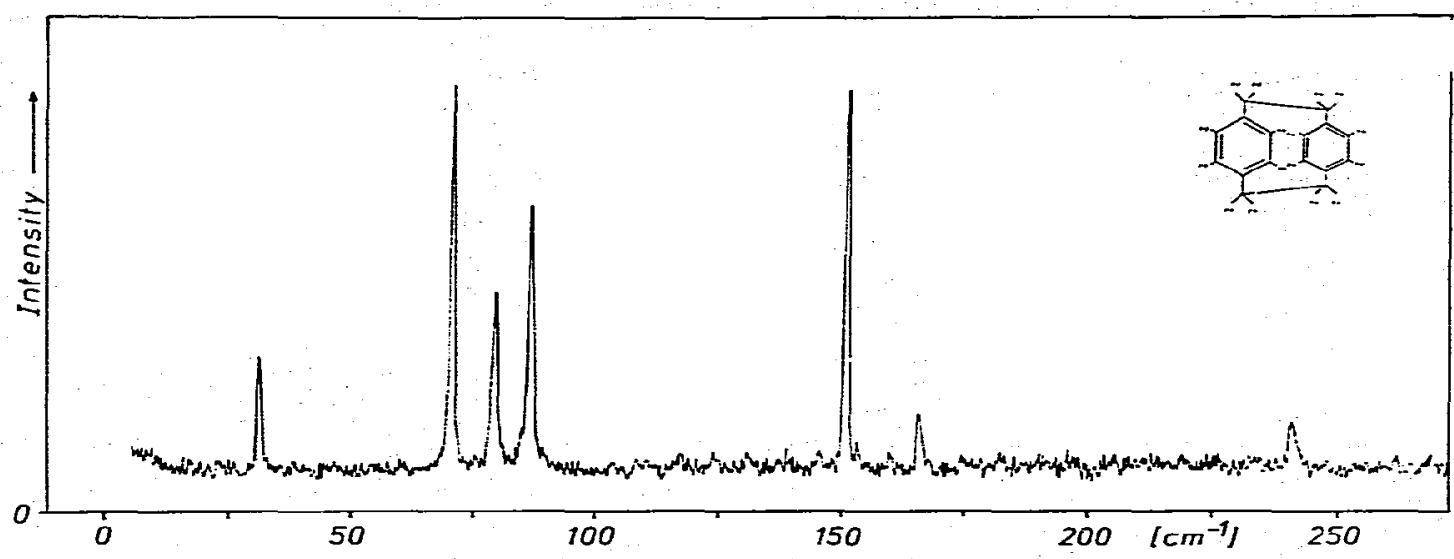

Fig. 5. Raman spectrum of a [2.2]paracyclophane single crystal (species A) at $5 \mathrm{~K}$. The incident light was polarized along the crystallographic $c$-axis.

ground state and can be described by the $D_{2}$ point group.

This result is in agreement with conclusions drawn from polarized absorption spectra, suggesting an $A_{u}$-type static distortion $[16,19-21]$.

\section{Discussion}

Having identified the prominent mode as a torsional vibration of the subunits, we now discuss the observed intensity distribution in terms of the theory of linear EPC in order to obtain information about the equilibrium shift under electronic excitation.

Because of existing reviews $[22,23]$, the derivation of the relative transition probabilities will not be repeated here. Essentially, using the BornOppenheimer approximation the Franck-Condon factors are calculated from the projection of the excited state nuclear eigenfunctions onto the ground state functions. This is particularly easy in the linear approximation, where these functions belong to harmonic potentials with equal eigenvalues but shifted along one normal coordinate with respect to each other.

Under these approximations, the nuclear potentials in the electronic states $u, l$ read as follows:

$E_{l}\left(Q_{u}\right)=E_{u l}+\left(2 M \hbar \omega_{l}^{3} S\right)^{1 / 2} Q_{u}+\frac{1}{2} M \omega_{l}^{2} Q_{u}^{2}$

$E_{u}\left(Q_{u}\right)=\frac{1}{2} M \omega_{1}^{2} Q_{u}^{2}$
By $l$, the ground state properties are characterized, $E_{u l}$ describes the energetic separation of the potential minima, $M$ is the reduced mass or moment of inertia of the particular normal mode with eigenvalue $\omega_{l}$. The vibrational eigenvalue $\omega_{l}$ is taken to be equal for the ground and excited state in accordance with the experimental finding. This justifies the neglect of higher terms in the expansion leading to eq. (2). The coupling strength $S$ is related to the well known dimensionless coupling parameter $a$ by [22]

$S=\frac{1}{2} a^{2}$

and can be used to calculate the normal coordinate shift $\Delta Q$ by

$\Delta Q=(2 S \hbar / \omega M)^{1 / 2}$.

The approximate shift $\delta$ of the intensity maximum to the 0,0 -line is given by

$\delta=S \hbar \omega$.

The spectral distribution $I(E)$ of the FC-factors can be expressed in a closed form and is reduced for low temperatures, i.e. for emission exclusively from the vibrationless electronic state, to the following simple expression [22]:

$$
\begin{aligned}
& I(E)=\sum_{m=0}^{m_{m}} \frac{e^{-s} S^{m}}{m !} \frac{(2 / \pi)^{1 / 2}}{E_{h w}} \\
& \quad \times \exp \left[-2\left(E_{u t}-E-m \hbar \omega\right)^{2} / E_{h w}^{2}\right]
\end{aligned}
$$


Assuming inhomogeneous broaciening, a gaussian lineshape with halfwidth $E_{\mathrm{hw}}$ was attributed to the individual lines. $E_{\mathrm{hw}}$ is determined experimentally. The summation limit $m_{m}$ prevents $E_{l}$ to exceed $E_{u}$. The vibrational quantum number is denoted by $m$.

The intensity distribution shown in fig. 3 is obtained by evaluating eq. ( 7 ) for a coupling strength $S=10$ and assuming a delta-function lineshape

The computer simulation of the emission spectra on the basis of this simple one-oscillator model only gave fair agreement and deviated considerably at the low energy side of the spectrum. This discrepancy could be removed to a large extent by considering in addition to the prominent dimer mode one monomer mode to be coupled to the electronic transition.

Intramolecular vibrations being prominent in monomer emission can often be ascribed to C-C stretching modes with an energy of about $1600 \mathrm{~cm}^{-1}$. This mode appears as the strongest vibronic transition in the phosphorescence spectrum of the "monomer" p-xylene [24].

The intensity function for this mode and its overtones can be described by linear EPC in the weak coupling range (i.e. $S=0.2-0.8$ ) for typical monomers [25].

The additional consideration of such a different coupled mode ${ }^{\dagger}$ leads to the following FC-distribution function:

$$
\begin{aligned}
& I(E)=\sum_{n=0}^{n_{m s}} \sum_{m=0}^{m_{m m}} \frac{\mathrm{e}^{-S_{c}} S_{c}^{n} \mathrm{e}^{-S_{z}} S_{2}^{m}}{n !} \\
& m ! \\
& \times \frac{(2 / \pi)^{1 / 2}}{E_{\mathrm{hw}}} \exp \left\{\frac{-2\left(E_{k t}-E-n h \omega_{c}-m h \hbar \omega_{\imath}\right)^{2}}{E_{\mathrm{hw}}^{2}}\right\}
\end{aligned}
$$

$n$ and $m$ being the vibronic quantum numbers of the $C-C-$ mode $\left(\omega_{c}\right)$ and the torsional mode $\left(\omega_{z}\right)$, respectively.

Using $S_{\mathrm{t}}=10, S_{\mathrm{c}}=0.25$ (huorescence) and $S_{\mathrm{t}}=13, S_{\mathrm{c}}=0.7$ (phosphorescence) the simulated spectrum fits the experimental one reasonably good, as depicted in fig. 2 . A constant half width $E_{\mathrm{hw}}=0.75 \hbar \omega_{\mathrm{r}}$ was used for the fit.

- IR spectra of [2.2] paracyclophane show lines in the range $1400-1600 \mathrm{~cm}^{-1}$ as well [25]. Since the fit is not particularly sensitive to the choice of $\omega_{n}$, the value measured for the monomer was used.
The variation of $S_{1}$ by \pm 1 leads to a clearly noticable deviation of the calculated emission maximum from the position of the observed one. The possible uncertainty of $S_{i}$ is therefore specified as \pm 0.5 . The accuracy of $S_{\mathrm{c}}$ as given in table 1 was estimated from the overall agreement in the long wavelength part of the spectrum. The calculated value of $S_{1}$ is not influenced by $S_{c}$, when this value is varied within its error limits.

The additionally observed low frequency modes $\left(v=50,80,180 \mathrm{~cm}^{-1}\right)$ are not included in the fit and the halfwidths used are chosen to give the best overall agreement with the experimental spectrum. This Ieads to the deviation of the calculated intensities for the first bands of the spectra, where the low energy modes are still experimentally resolved.

The decreasing resolution in the experimental spectra at lower energies is presumably due to additional vibronic bands of higher energies leading to destructive interference.

As listed in table 1, the coupling strengths obtained from the fit are similar for all species. The value $S=10$ obtained for the fluorescence spectra does not exclude the detection of the 0,0-transition. As can be seen in fig. 2, a weak emission can be detected at the position of the first absorption band (the wavenumbers are equal within $10 \mathrm{~cm}^{-1}$ ). The intensity ratio of the 0,0 and 0,10 band confirms our interpretation of the spectrum with $S=10$.

In contrast, because of $S=13$ in the $T_{1} \rightarrow S_{0}$ transition, the 0,0 -band is no longer detectable. The first observable band is the 0,2-transition.

As coupling strength $S$, mode frequency $\omega_{t}$ and normal coordinate are known for the torsional mode, the shift of the equilibrium position can be calculated from eq. (5). The shift $\Delta Q$ relative to the<smiles>C#Cc1ccc(C)cc1</smiles>

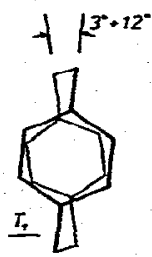

Fig. 6. Torsional mode equilibrium positions of the paracyclophane subunits in the $I_{1}$ and $S_{0}$ electronic states (seen along the benzene out of plane axis). 
ground state position, which in turn is twisted by approximately $3^{\circ}$ from $D_{2 n}$-symmetry, amounts to $10.6^{\circ}$ in the $S_{1}$ state and $12^{\circ}$ in $T_{1}$ (see fig. 6).

The accuracy of the equilibrium shift $\Delta Q$ is mainly determined by the error of $S_{\mathrm{t}}$ (see table 1).

\section{Conclusion}

The fluorescence and phosphorescence spectra of paracyclophane show an intensity distribution which is similar to the spectra of other phanes. Because of a partially resolved vibronic structure we were able to identify the prominent coupled mode as a dimer vibration. The strong coupling of this mode to the electronic transition is proof of a relatively large equilibrium shift under electronic excitation. Such a shift has to be expected, if the electronic density distribution in the molecule, especially along the out-of-plane axis, is changed in the excited states. An increase of out-of-plane orbital dimensions was postulated and used earlier for an interpretation of phane spectra [9].

Assuming similar force constants for dimer modes of different phanes, the increased reduced mass for nearly all phanes investigated will lead to a lower mode frequency. In many cases the vibrational structure will then not be resolvable. However, the general spectral appearance will be unchanged and will depend only on $S$ and $\omega$. The red shift $\delta$ of the peak intensity from the 0,0 -transition is given by $\delta \approx S \omega$ and can easily amount to $\delta=2000-$ $3000 \mathrm{~cm}^{-1}$. The consideration of this shift is particularly important; when discussing monomer/ dimer optical red shifts. A quantitative evaluation, however, is only possible, when the coupling is due to one mode only, of which the energy can be determined, as in the case of paracyclophane.

\section{Acknowledgernent}

We like to thank Mr. H. Zimmermann for growing the paracyclophane single crystals. We are especially grateful to Mr. H. Fischer for the gift of the different, isotopically labelled molecules.

* Recent experiments on [2.2] $(2,3,5,6)$ tetramethylparacyclophane show a vibrational progression similar to the one observed in paracyclophane [26].

\section{References}

[1] J.3. Markham, Rev. Mod. Phys. 31 (1959) 956

[2] J.T. Ritter and J.J. Markham, Phys. Rev. 185 (1969) 1201 .

[3] Muto, Progr. Theoret. Phys. (Kyoto) 4 (1949) 181.

[4] K. Huang and A. Rhys, Proc. Roy. Soc. London $204 \mathrm{~A}(1950) 406$.

[5] S.I. Pekar, Zh. Eksp. Teor. Fiz 20 (1950) 510; Usp. Fiz. Nauk 50 (1953) 197.

[6] M. Lax, Photoconductivity Conference, Atlantic City, 1954, eds. R.G. Breckenridge, B.R. Russell and E.E. Hahn (Wiley, New York, 1956) p. 111.

[7] D. Curie, C.R. Acad. Sci. Paris 246 (1958) 404.

[8] D. Haarer, J. Chem. Phys. 67 (1977) 4076.

[9] D. Schweitzer, J.P. Colpa, K.H. Hausser, M. Haenel and H.A. Staab, J. Luminesc. 12/13 (1976) 363; D. Schweitzer, J.P. Colpa, J. Behnke, K.H. Hausser, M. Haenel and H.A. Staab, Chem. Phys. 11 (1975) 373.

[10] D. Schweitzer, K.H. Hausser. V. Taglieber and H.A. Staab Chem. Phys. 14 (1976) 183.

[11] W. Goldacker, D. Schweitzer, K.H. Hausser and H.A. Staab J. Luminesc. 18/19 (1979) 415.

[12] J.P. Colpa, K.H. Hausser and D. Schweitzer, Chem. Phys. 29 (1978) 187.

[13] G. Melzer, D. Schweitzer, K.H. Hausser, J.P. Colpa and M.W. Haenel, Chem. Phys. 39 (1979) 229.

[14] K.P. Dinse and C. von Borezyskowski, Chem. Phys. 44 (1979) 93.

[15] W. Goldacker, Thesis, University Heidelberg, W. Germany 1979.

[16] A. Ron and O. Schnepp, J. Chem. Phys. 37 (1962) 2540.

[17] D.K. Lonsdale, H.J. Milledge and K.V. Krishna Rao, Proc. Roy. Soc. A255 (1960) 82 .

[18] H. Hope and J. Bernstein and K.N. Trueblood, Acta Cryst. B28 (1972) 1733.

[19] M.I. Vala, I.H. Hillier, S.A. Rice and J.J. Jortner, J. Chem. Phys, 44 (1966) 23.

[20] L.H. Hillier, L Glass and S_A. Rice, J. Chem. Phys. 45 (1966) 3015

[21] A. Ron and O. Schnepp, J. Chem. Phys. 44 (1966) 19.

[22] T.H. Keii, Phys. Rev. 140A (1965) 601.

[23] J.L. Richards and S.A. Rice, J. Chem. Phys. 54 (1971) $20 \mathrm{~F} 4$.

[24] Ph.J. Vergragt, J.A. Kooter and J.H. van der Waals, Mol. Phys. 33 (1977) 1523.

[25] W. Goldacker, unpublished results.

[267 G. Melzer. unpublished results. 